\title{
Research on vibration and acoustic radiation characteristics of underwater vehicle superstructure with composite sandwich panel
}

\author{
Di Jia ${ }^{1, ~ a, ~ X u e r e n ~ W a n g ~}{ }^{1, ~ b}$, Ruijie Liu ${ }^{1, c}$, Xuhong Miao ${ }^{1, d}$ \\ ${ }^{1}$ Naval Academy of Armament, Beijing 100161 \\ ajiadi2017@aliyun.com, b'wangxueren@aliyun.com, \\ cliuruijie@aliyun.com, ${ }^{\mathrm{d}}$ miaoxuhong@aliyun.com
}

Keywords: underwater vehicle; sandwich panel; superstructure; vibration acoustic radiation

Abstract: Compared with traditional steel materials, sandwich panel has been widely applied in underwater vehicle structure because of the high specific strength, excellent acoustic performance and strong design ability. Based on the acoustical-solid coupling finite element method, the vibration and acoustic radiation characteristics of underwater vehicle superstructure are analyzed in this paper. On this basis, the effect rule of shell thickness parameters on the vibration and acoustic radiation characteristics of underwater vehicle superstructure is explored, and the acoustic optimization design plan of superstructure with sandwich panel is proposed, so as to provide reference for the acoustic design of superstructure.

\section{Introduction}

The sandwich panel has excellent mechanical properties, such as high strength, high rigidity and fatigue resistance, which can greatly reduce the weight of the underwater vehicles, it can reduce the center of gravity and increasing the speed while satisfying the mechanical performance requirements [1]. Superstructure is an important part of underwater vehicles, compared with the traditional steel material, when sandwich panel used in the underwater vehicles superstructure, the effect of vibration and acoustic radiation performance of the underwater structure of the underwater acoustic performance is the focus of the study. At present, the superstructure of the composite material mostly adopts the sandwich panel structure, which is mainly composed of two thin fiber skins and a middle thicker low density material. The structure of the sandwich plate is simple and the preparation technology is mature, it has been widely used [2]. At the same time, the mechanical properties of the sandwich panel structure are simple, a variety of dynamic and static mechanical properties have been widely studied, such as in-plane extrusion, shear, out of plane bending deformation, fatigue, vibration and noise and explosion impact [3,4]. Meanwhile, the effect of the thickness of skin layer, the order of the plies, the angle of ply and the thickness and material of the material on the mechanical properties of the sandwich structure [5,6] were also studied. Therefore, this article takes underwater vehicle structure as the research object, the composite stiffened sandwich structure used in the superstructure, based on the acoustic-mechanical coupling finite element method to carry out structural underwater acoustic radiation characteristics analysis, found the characteristics of composite materials on the superstructure underwater acoustic radiation characteristics of the law, it provides a reference for low noise design of underwater vehicle structure.

\section{Theoretical formulations}

According to the acoustic theory, the acoustic wave equation in the flow field is as follows:

$$
\nabla^{2} p=\frac{1}{c^{2}} \frac{\partial^{2} p}{\partial t^{2}}
$$

where $p$ is the instantaneous sound pressure, $t$ is the time variable, $c$ is the speed of sound, $\nabla^{2}$ is Laplacian operator. 
The fluid is discretized into numbers of finite elements, the sound pressure and particle velocity at any point in the element and the derivatives of the time can be calculated by the pre-defined shape function that the sound pressure and particle velocity on the element node, in the last formula, the variation of sound pressure is approximated, and the finite element equation matrix of the sound field in the fluid region can be obtained as follows:

$$
\boldsymbol{M}_{f} \boldsymbol{P}_{+} \boldsymbol{K}_{f} \boldsymbol{P}+\rho_{f} \boldsymbol{R} \boldsymbol{E}=\mathbf{0}
$$

where $\boldsymbol{M}_{f}$ is the fluid equivalent mass matrix, $\boldsymbol{K}_{f}$ is the fluid equivalent stiffness matrix, $\boldsymbol{R}$ is the fluid-structure coupling matrix, and $\boldsymbol{\&}$ is the second derivative of the unit node displacement $\boldsymbol{U}$ versus time, $\boldsymbol{O}$ is a zero matrix.

Equation (2) needs to be introduced into the acoustic absorption term when there is a damping sound absorption material at the boundary of the fluid region and at the interface between the fluid and the structure. The damping matrix equation can be obtained by derivation similar to the previous one:

$$
\boldsymbol{M}_{f} \boldsymbol{E} \boldsymbol{C}_{f} \boldsymbol{E}+\boldsymbol{K}_{f} \boldsymbol{P}+\rho_{f} \boldsymbol{R}=[0] .
$$

where $\boldsymbol{C}_{f}$ is the acoustic damping matrix.

It exists the interaction between the fluid and the structure at their interface when the structure is placed in the fluid medium. According to the principle of virtual work, the facial force can be transferred to the element node equivalently, so we can get the coupled vibration matrix equation of structure and flow field as follow:

$$
M_{s} \boldsymbol{E}_{+} \boldsymbol{C}_{s} \boldsymbol{Q}_{+} \boldsymbol{K}_{s} \boldsymbol{U}=\boldsymbol{F}_{s}+\boldsymbol{F}_{f} \text {. }
$$

where $\boldsymbol{M}_{s}, \boldsymbol{K}_{s}, \boldsymbol{C}_{s}$ represent the mass matrix, stiffness matrix and damping matrix of the structure, respectively. $\boldsymbol{F}_{s}$ is the load vector of the structure, $\boldsymbol{F}_{f}=\boldsymbol{R}^{T} \boldsymbol{P}$.

By substituting equations (3) and (4), it can be summed up in a matrix form as follows:

$$
\left(\begin{array}{ll}
\boldsymbol{M}_{s} & {[0]} \\
\boldsymbol{\rho}_{f} \boldsymbol{R} & \boldsymbol{M}_{f}
\end{array}\right)\left\{\begin{array}{l}
\boldsymbol{H} \\
{[0]}
\end{array}\right\}+\left(\begin{array}{ll}
\boldsymbol{C}_{s} & {[0]} \\
{[0]} & \boldsymbol{C}_{f}
\end{array}\right)\left\{\begin{array}{l}
\boldsymbol{\Theta} \\
\boldsymbol{E}
\end{array}\right\}+\left(\begin{array}{cc}
\boldsymbol{K}_{s} & -\boldsymbol{R}^{T} \\
{[0]} & \boldsymbol{K}_{f}
\end{array}\right)\left\{\begin{array}{l}
\boldsymbol{U} \\
\boldsymbol{P}
\end{array}\right\}=\left\{\begin{array}{l}
\boldsymbol{F}_{s} \\
{[0]}
\end{array}\right\} .
$$

According to the discretized matrix equation (5), which is the coupling between superstructure and fluid in the composite, the displacement at the surface of the structure and the pressure in the flow field can be obtained.

\section{Research on vibration and acoustic radiation characteristics of underwater superstructure with composite sandwich panel}

Numerical Analysis of the establishment of the model. The underwater vehicle structure is mainly composed of the main structure of steel cabin and the superstructure shell structure of composite material, of which the cabin structure is a double-shell structure composed of stiffeners and steel frame, the length of the cabin structure $L=5.4 \mathrm{~m}$, rib spacing $l=0.6 \mathrm{~m}$, the shell diameter $D=4.3 \mathrm{~m}$, the inner shell diameter $d=2.8 \mathrm{~m}$, from the cabin structure of bow to stern end, the distance between any two adjacent steel frames is $\Delta l=1.8 \mathrm{~m}$, and the pillar with the size of $\Phi 46^{*} 12$ under the crossing position of the steel frame is used as the supporting member. A schematic diagram of the superstructure of composite materials is shown in Figure 1. 


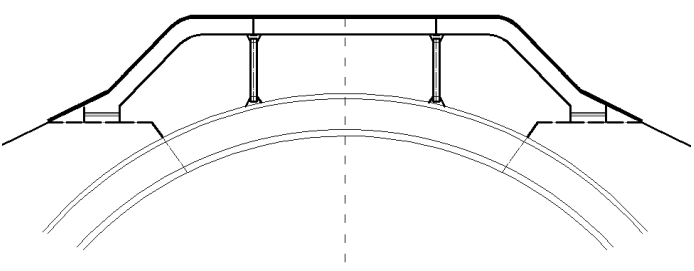

( a ) Superstructure diagram

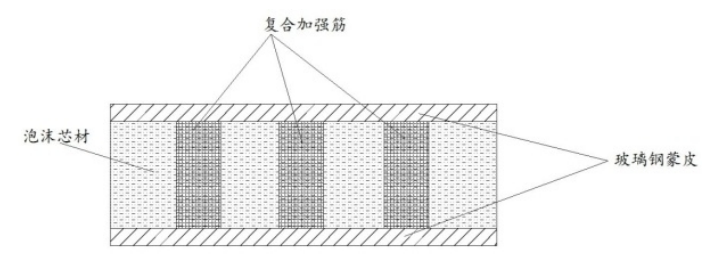

( b ) Sandwich panel structure reinforced with composite materials diagram

Figure 1 .Composite materials superstructure diagram

Due to the sandwich panel structure reinforced with composite materials, the performance of different layered materials plays a decisive role in the results. Table 1 shows the performance parameters of composite materials superstructure.

Table 1.composite materials performance parameters of each layer ${ }^{[2][7]}$

\begin{tabular}{|c|c|c|c|c|}
\hline Name & Steel & GFRP & PVC & $\begin{array}{c}\text { Common } \\
\text { stiffeners(T300/3234) }\end{array}$ \\
\hline $\operatorname{Density}\left(\mathrm{kg} / \mathrm{m}^{3}\right)$ & 7850 & 1780 & 450 & 790 \\
\hline \multirow{3}{*}{ Elastic Modulus(Gpa) } & \multirow{3}{*}{210} & $E_{x}=12.0$ & \multirow{3}{*}{0.08} & $E_{x}=128$ \\
\hline & & $E_{y}=10.0$ & & $E_{y}=8.7$ \\
\hline & & $E_{z}=6.0$ & & $E_{z}=8.7$ \\
\hline \multirow{3}{*}{ Shear modulus(Gpa) } & \multirow{3}{*}{-} & \multirow{3}{*}{$G_{x y}=G_{x z}=G_{y z}=2.5$} & \multirow{3}{*}{-} & $G_{x y}=4.0$ \\
\hline & & & & $G_{x z}=4.0$ \\
\hline & & & & $G_{y z}=4.0$ \\
\hline \multirow{3}{*}{ Poisson's ratio } & \multirow{3}{*}{0.3} & $P R_{x y}=0.15$ & \multirow{3}{*}{0.2} & $P R_{x y}=0.32$ \\
\hline & & $P R_{x z}=0.2$ & & $P R_{x z}=0.32$ \\
\hline & & $P R_{y z}=0.15$ & & $P R_{y z}=0.3$ \\
\hline Structural damping & 0.0005 & 0.06 & 0.02 & 0.03 \\
\hline
\end{tabular}

Study on vibration and acoustic radiation characteristics of underwater superstructure with composite materials. This section analyzes the material parameters of steel, acoustic materials and composite materials, in which the thickness of the steel superstructure shell thickness is $5 \mathrm{~mm}$, and the acoustic material scheme is to lay acoustic materials on the steel superstructure, considering the mass effect of the acoustical material, the areal density of the acoustic material is $28.6 \mathrm{~kg} / \mathrm{m}^{2}$, while the thickness ratio program of the composite materials superstructure is 6/32/6. Acoustic materials and composite materials superstructure are shown in Figure 2- Figure 3.

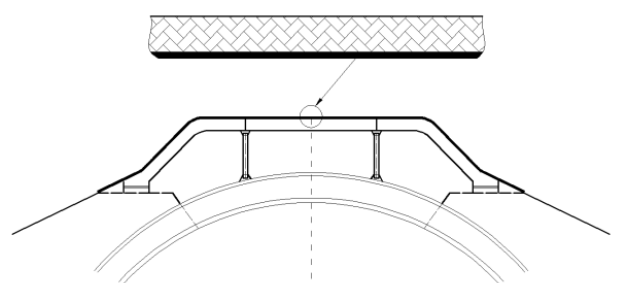

Figure 2. Acoustic materials superstructure

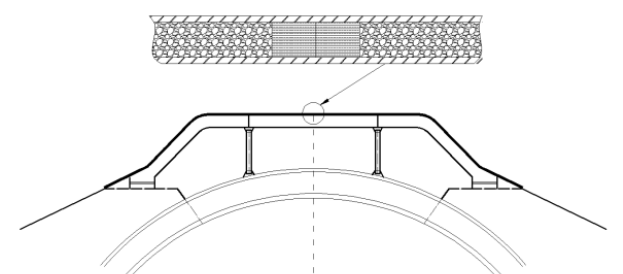

Figure 3. composite materials superstructure

In this section, vertical unit concentrated force is applied to the center of the superstructure shell, and the mean square vibration velocity and sound source level of the shell surface are adopted as the assessment parameters of structural underwater vibration and radiation noise. Figure 4 shows the variety curve which the vibration and acoustic radiation characteristics of underwater superstructure with different materials. 


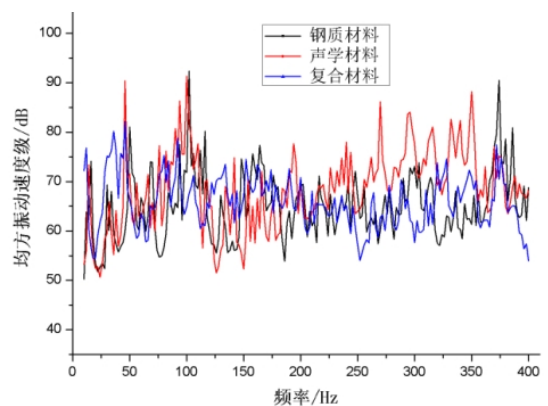

(a)The velocity level contrast curve of the mean square vibration

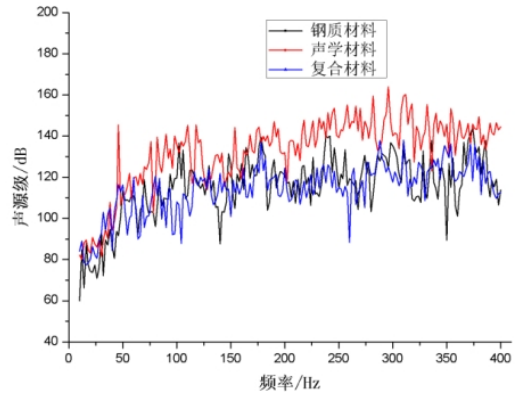

(b)Sound source level comparison curve

Figure 4. Contrast curves of vibration and acoustic radiation characteristics of underwater superstructure

It can be seen from Figure 4, the underwater acoustic radiation characteristics of the superstructure change obviously when different materials are used. in the frequency range of $200 \mathrm{~Hz}-400 \mathrm{~Hz}$, the level of underwater vibration of acoustics superstructure level is high, and the level of underwater radiation noise are the largest in the entire frequency range. The noise level of underwater vibration of composite superstructure is lower than steel superstructures in certain frequency range. Overall, the level of underwater radiation noise is low when the structure of superstructure is used, and the level of radiation noise is higher when using acoustic materials.

In order to quantify the calculation results better, the total band-level situation of the underwater acoustic vibration characteristics of the three superstructures is shown in Table 2.

Table2. The total band-level situation of the underwater acoustic vibration characteristics $(d B)$

\begin{tabular}{|c|c|c|c|c|c|c|}
\hline \multirow{2}{*}{$\begin{array}{l}\text { frequency } \\
\operatorname{band}(H z)\end{array}$} & \multicolumn{3}{|c|}{$\begin{array}{l}\text { The mean square vibration velocity } \\
\text { level on the surface of the shell plate }\end{array}$} & \multicolumn{3}{|c|}{ The level of Sound source } \\
\hline & Steel & $\begin{array}{l}\text { Acoustic } \\
\text { material }\end{array}$ & Composites & Steel & $\begin{array}{l}\text { Acoustic } \\
\text { material }\end{array}$ & Composites \\
\hline 10-100 & 92.6 & 98.3 & 92.2 & 130.9 & 152.3 & 130.6 \\
\hline $100-200$ & 96.6 & 96.0 & 88.9 & 148.1 & 158.2 & 142.7 \\
\hline $200-300$ & 86.7 & 95.2 & 84.6 & 150.3 & 170.6 & 144.4 \\
\hline $300-400$ & 95.4 & 96.7 & 88.5 & 152.5 & 169.3 & 148.4 \\
\hline $10-400$ & 99.6 & 102.0 & 95.3 & 155.4 & 173.2 & 150.7 \\
\hline
\end{tabular}

It can see from Table 2 that the influence of material parameters on underwater acoustic radiation characteristics of superstructure is different in different frequency bands. Compared with the steel superstructure, in the frequency range of $10 \mathrm{~Hz}-400 \mathrm{~Hz}$, when the superstructure adopts composite material, the mean square vibration velocity on the shell surface decreases $4.3 \mathrm{~dB}$ and the total sound source level decreases $4.7 d B$; When the superstructure uses acoustic materials, the mean square vibration velocity of the shell surface increases $2.4 d B$ and the total sound source level increases $17.8 d B$.

\section{Study on the influence of shell thickness parameters on the underwater acoustic vibration characteristics}

Composites shell structure of the superstructure is a typical stiffened sandwich structure, the thickness of the upper and lower GFRP surface layer is $6 \mathrm{~mm}$, the thickness of the middle layer of the PVC foam core layer is $32 \mathrm{~mm}$, the width of the ribs set to $60 \mathrm{~mm}$ ordinary stiffener structure, the general stiffener structure extends over the entire composites superstructure shell structure. As an important factor to influence the underwater acoustic radiation characteristics of composites superstructure, it includes two important parameters: the thickness of the shell slab and the thickness of 
the slab core. Therefore, in this section, the reference structure of composites superstructure is taken as the research object. The influence of the thickness parameters of the shell plate on the underwater acoustic radiation characteristics of the composite superstructure is studied by changing the shell thickness and the core thickness.

The parameters of surface thickness. This section takes the composites superstructure as the research object, on the basis of stable thickness of the core layer, there are three kinds of thickness, the thickness of the surface layer is $4 \mathrm{~mm}, 5 \mathrm{~mm}$ and $6 \mathrm{~mm}$, the vibration and acoustic vibration characteristics of underwater were studied respectively.

Under the vertical unit force in the center of the superstructure, the mean square vibration velocity of the wet surface of the shell structure and the sound source level contrast curve are shown in Figure 5.

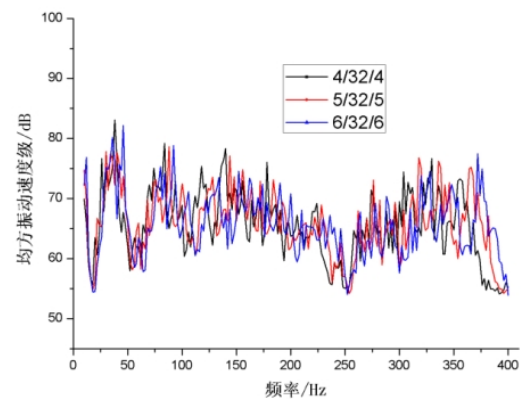

(a)The velocity level contrast curve of the mean square vibration

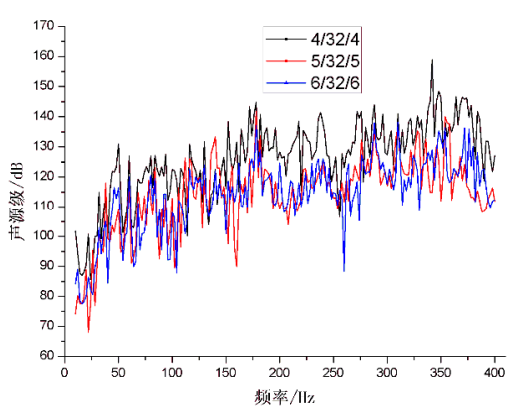

(b)Sound source level comparison curve

Figure 5. Contrast curves of vibration and acoustic radiation characteristics of underwater superstructure

As shown in Figure 5, the trend of the mean square vibration velocity and the sound source level vary with the frequency is basically the same that in the case of different surface thickness of the superstructure surface. With the thickness of the shell increase, the stiffness of the shell increase and the mean square vibration velocity of the surface shifts to the right, the level of sound source also decrease.

In order to quantify the calculation results better, Table 3 shows the case of vibration and acoustic radiation characteristics of underwater vehicle superstructures with changing the thickness of the layer in different frequency bands.

Table3. Frequency range of vibration and acoustic radiation characteristics of underwater $(d B)$

\begin{tabular}{c|c|c|c|c|c|c}
\hline \multirow{2}{*}{$\begin{array}{c}\text { Frequency } \\
\text { band }(\boldsymbol{H z})\end{array}$} & \multicolumn{2}{|c|}{$\begin{array}{c}\text { The mean square vibration velocity } \\
\text { level on the surface of the shell }\end{array}$} & \multicolumn{3}{|c}{ Sound source level } \\
\cline { 2 - 7 } & $\mathbf{4 / 3 2 / 4}$ & $\mathbf{5 / 3 2 / 5}$ & $\mathbf{6 / 3 2 / 6}$ & $\mathbf{4 / 3 2 / 4}$ & $\mathbf{5 / 3 2 / 5}$ & $\mathbf{6 / 3 2 / 6}$ \\
\hline $10-100$ & 92.2 & 91.7 & 90.9 & 131.5 & 131.4 & 130.6 \\
\hline $100-200$ & 90.2 & 89.5 & 88.8 & 143.5 & 143.5 & 142.7 \\
\hline $200-300$ & 85.6 & 84.6 & 84.2 & 145.3 & 144.8 & 144.4 \\
\hline $300-400$ & 91.6 & 90.2 & 88.5 & 151.7 & 150.6 & 148.4 \\
\hline $10-400$ & 96.5 & 95.3 & 95.1 & 152.6 & 151.8 & 151.1 \\
\hline
\end{tabular}

As shown in table 3: in the frequency range of $10 \mathrm{~Hz}-400 \mathrm{~Hz}$, with the thickness of the superstructure surface layer increase, the level of vibration and acoustic radiation characteristics of underwater is decreasing. When the thickness of the surface layer increasing from $4 \mathrm{~mm}$ to $6 \mathrm{~mm}$, the mean square vibration velocity of the shell plate decreases $1.4 d B$, and the sound source level decreases $1.5 d B$. The change of acoustic radiation characteristics in the frequency range of $100 \mathrm{~Hz}-300 \mathrm{~Hz}$ is significantly, especially in the frequency range of $300 \mathrm{~Hz}-400 \mathrm{~Hz}$, the mean square vibration velocity of the shell surface decreases $3.1 \mathrm{~dB}$, and the sound level decreases $3.3 \mathrm{~dB}$. 
The parameters of core thickness. In this section, the basic structure of the superstructure of composite materials is taken as the research object. Three kinds of core layer thickness are setting without changing the thickness of the surface layer $(6 \mathrm{~mm})$, the thickness of the core layer is set as $32 \mathrm{~mm}, 36 \mathrm{~mm}$ and $40 \mathrm{~mm}$. The vibration and acoustic radiation characteristics of underwater about three structures were analyzed respectively.

Under the vertical unit force in the center of the superstructure, the mean square vibration velocity of the wet surface of the shell structure and the sound source level contrast curve are shown in Figure 6.

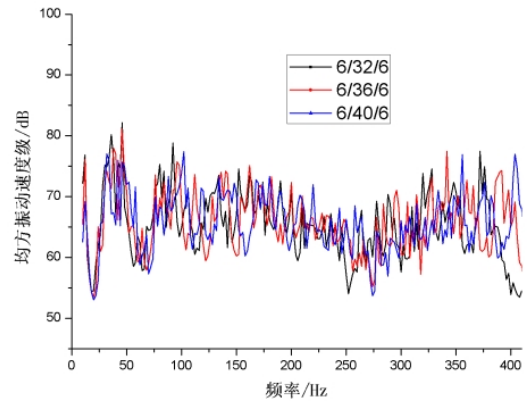

(a)The velocity level contrast curve of the mean square vibration

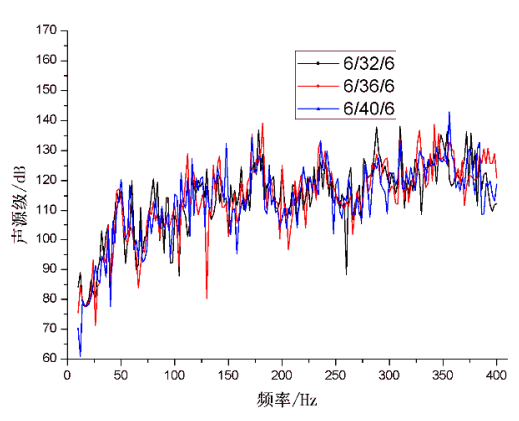

(b)Sound source level comparison curve

Figure 6. Contrast curves of vibration and acoustic radiation characteristics of underwater superstructure

As shown in Figure 6, the trend of the curve that vibration and acoustic radiation characteristics of underwater superstructure have little changing with the thickness of the superstructure core layer changing. With the increase of the shell core thickness, the stiffness of the shell structure increases, it makes the curve shift to the right, while there is no significant difference between the sound source level curves. In order to better quantify the calculation results, Table 4 shows in the different frequency bands, the Influence of vibration and acoustic radiation characteristics of underwater superstructure with core thickness changing.

Table4. Frequency range of vibration and acoustic radiation characteristics of underwater $(d B)$

\begin{tabular}{c|c|c|c|c|c|c}
\hline \multirow{2}{*}{$\begin{array}{c}\text { Frequency } \\
\text { band }(\boldsymbol{H z})\end{array}$} & \multicolumn{2}{|c|}{$\begin{array}{c}\text { The mean square vibration velocity level on the } \\
\text { surface of the shell }\end{array}$} & \multicolumn{3}{c}{ Sound source level } \\
\cline { 2 - 7 } & $\mathbf{6 / 3 2 / 6}$ & $\mathbf{6 / 3 6 / 6}$ & $\mathbf{6 / 4 0 / 6}$ & $\mathbf{6 / 3 2 / 6}$ & $\mathbf{6 / 3 6 / 6}$ & $\mathbf{6 / 4 0 / 6}$ \\
\hline $10-100$ & 90.9 & 89.8 & 88.2 & 130.6 & 127.0 & 128.7 \\
\hline $100-200$ & 88.8 & 89.1 & 89.4 & 142.7 & 145.5 & 142.7 \\
\hline $200-300$ & 84.2 & 85.3 & 84.9 & 144.4 & 143.1 & 141.8 \\
\hline $300-400$ & 88.5 & 88.9 & 87.2 & 148.4 & 148.7 & 148.9 \\
\hline $10-400$ & 95.1 & 94.5 & 93.2 & 151.1 & 150.7 & 150.5 \\
\hline
\end{tabular}

It is known from table 4 that: in the frequency range of $10 \mathrm{~Hz}-400 \mathrm{~Hz}$, the radiation noise characteristics of underwater composites superstructure decreasing with the increase of the thickness of the core layer. When the core thickness increases from $32 \mathrm{~mm}$ to $40 \mathrm{~mm}$, the mean square vibration velocity of the shell surface decreases $1.9 d B$ and the sound source level decreases $0.6 d B$. However, in the frequency range of $10 \mathrm{~Hz}-100 \mathrm{~Hz}$, the radiation noise characteristics of underwater changes obviously with the increase of the shell core thickness. The mean square vibration velocity on the shell surface decreases $2.7 d B$ and the total sound level decrease $1.9 d B$.

\section{Conclusions}

Compared with traditional steel superstructure, the changing of material properties will have a great influence on vibration and acoustic radiation characteristics of underwater after the underwater vehicle 
superstructure adopting composite sandwich panel. Based on the acoustic structure coupling finite element analysis technology, this paper analyzed vibration and acoustic radiation characteristics of underwater vehicle superstructure with composite sandwich panel, and the influence rule of shell thickness parameters is further explored. The following conclusions are obtained:

1) When the superstructure adopts composite sandwich panel, the acoustic radiation characteristics of the superstructure are much better than that of the steel material; when the superstructure adopts steel material, the acoustic radiation characteristics of the superstructure are much better than that of the Acoustic material. So, it is benefit that the underwater vehicle superstructure adopt composite sandwich panel.

2) It is benefit that increasing the thickness of the shell to improve the performance of vibration and acoustic radiation characteristics of underwater vehicle superstructure. Increasing the thickness of the surface is beneficial to improve the acoustic performance of the structure at high frequency $(300 \mathrm{~Hz}-400 \mathrm{~Hz})$, while increasing the core thickness can improve the acoustic performance of the structure at low frequency $(10 \mathrm{~Hz}-100 \mathrm{~Hz})$.

\section{Acknowledgements}

This work was financially supported by The National key research and development program of China(2016YFC0303406),Assembly Advanced Research Fund Of China(6140210020105),Major innovation projects Of High Technology Ship Funds of Ministry of Industry and Information of P.R.China, National Natural Science Foundation China (No.51209052),High Technology Ship Funds of Ministry of Industry and Information Technology of P.R.China, Fundamental Research Funds for the Central Universities(HEUCFD1515、HEUCFM170113),China Postdoctoral Science Foundation (2014M552661).

\section{References}

[1]Jiang Qian,Nan Li, Wenqiang Shi. The application and development of composites for foreign naval warships' superstructure [J].JIANCHUAN KEXUE JISHU, 2015(1):233-237.

[2]Wei Wang. Investigation of Joint between Lattice Composite Superstructure and Steel Main Hull and Design. Journal of Harbin Engineering University, 2016.

[3]Xuewen Yin.Study on vibration and acoustic radiation from (laminatated composite) cylindrical shells. Journal of ShangHai Jiao Tong University, 2008.

[4]Li X W, Li P, Lin Z, et al. Mechanical Characterizations of Composite Sandwich to Steel Joint for Marine Structures[C]. Materials Science Forum. 2015, 813: 210-219.

[5]Li X, Li P, Lin Z, et al. Mechanical behavior of a glass-fiber reinforced composite to steel joint for ships [J]. Journal of Marine Science and Application, 2015, 14(1): 39-45.

[6]Fotsing E R, Leclerc C, Sola M, et al. Mechanical properties of composite sandwich structures with core or face sheet discontinuities [J]. Composites Part B: Engineering, 2016, 88: 229-239.

[7] Yang N, Wei W, Dong Y, et al. Progressive damage analysis of steel-to-composite joints of radome[J]. Journal of Harbin Engineering University, 2014, 35(10):1183-1188 and 1235. 